\title{
Tibetan Women's Poverty and Unmarried Birth from the Perspective of
}

\section{Accurate Poverty Alleviation}

\author{
Fan Yang ${ }^{1,2}$, Tianhui Zhuang ${ }^{2}$, Weizhong Zeng ${ }^{2}$ \\ ${ }^{1}$ School of Management, Sichuan Agricultural University, Chengdu, 611130, China \\ ${ }^{2}$ Southwest Center for Poverty Alleviation and Development Research, Chengdu, 611130, China
}

Keywords: Tibetan, Unmarried birth, Women poverty, Alleviate supporting poverty

Abstract: Unmarried birth is the helpless and inefficient choice of Tibetan women when facing marriage squeeze and survival pressure. It can easily lead to economic poverty by raising children alone and causing poor health conditions for labor intensity damage, losing the development opportunity for low-level redundant labors and hard ascended social capitals, resulting emotional crisis that can't remarried, thus reducing the welfare of women. The intrinsic connection between out of unmarried births and women's poverty can be blocked through institutional arrangements, policy support and cultural guidance. In the political background of accurate support poverty, precise poverty alleviation and forming a comprehensive well-off society vigorously promoted by our country, properly solve this problem has a historic cultural significance.

\section{Introduction}

Unmarried birth refers to fertility behavior occurred outside the legal marriage. Since the 1960s, the unmarried birth phenomenon in Western County keep increasing and forming a unique and important social structure so far. Unmarried birth and female welfare problems caused by it has gradually become the focus in domestic and foreign academic circles. Numerous studies of foreign scholars showed that unmarried birth is closely connected with female welfare, including financial hardship, damaging women's health, forming marriage barriers and others which greatly decreased their welfares. Unmarried birth phenomenon has a long history in Tibetan areas, and in fact, retained until today. The investigation of the relationship between female unmarried birth and women poverty is not only the focus of sociological gender studies, but also given a new era significance under the background of the practice of accurate supporting poverty and alleviating poverty.

\section{Estimate the size of Tibetan female unmarried birth}

No matter how the form of marriage is, the fertility behavior of Tibetan is carried out basically in a state of natural without interventions which the existence and manifestation of this mode is also relatively common even in modern agricultural and pastoral areas. In this natural fertility patterns, there indeed exists certain unmarried birth phenomenon in Tibet. The female who is not married or marring males is called "Maoshenjiang” (female bachelor) in Tibetan. In Qinghai Tibetan areas, generally girls will hold a "Dai Tian Tou” ceremony when they are fifteen or sixteen years old, after which will have a legal identity on sexual life, thus, the phenomenon of premarital sex and unmarried birth is more prevalent in this region, and the situation of having children without married, having family without husband is common. According to the data provided in the study on modern marriage and family by Lei Mingguang, there are 76 females who given unmarried births in 
certain tribe of Naqu country Tibet province in recent years, accounting for $17 \%$ of the total population. The sample survey of Tibet University in 1988 has showed that the perception of unmarried birth in Tibet pastoral areas is $6.7 \%$, and in rural areas is slightly lower at $4.0 \%$. Wang Wenchang once randomly researched the marriage structure of 11 families in east Tibet, the counties like Changdu, Dingqing, Leiwuqi, Chaya and others, among which two families belongs to single-parent family formed by unmarried birth, accounting 18.18\%. The survey on 379 females aged 15-64 in remote pastoral areas of Qinghai Province by the Institute of Population Research, Peking University in 2009 shows that the number of unmarried female (not including married but separated, divorced and widowed) but having children accounted 6.1\%. From the survey of 23 families established filing cards in Laza rustic Benge village Litang county Ganzi Autonomous Prefecture Sichuang province in August 2015, the author found that four of them belong to single-parent family which is formed by unmarried birth, accounting $17.39 \%$.

\section{Effect mechanism of Tibetan female unmarried birth}

Any phenomenon has its inherent logic. Tibetan women's choice of unmarried fertility is influenced by nature, religion, economy, culture and other multiple factors.

Unmarried birth is the helpless choice of Tibetan women facing marriage squeeze. The marriage squeeze of Tibetan female comes from three aspects, one is polyandry policy, second is religious caused the number of marriage male decreased, and third is the slow development of outside marriage. From the aspect of marriage morphology, Tibetan polyandry has a long tradition and its forming is the nature choice when facing isolation and cold geographic environment and adapting to the regional economic bases. It presents the rationality of seeking to maximize the benefits of family in a particular environment which causing the phenomenon of marriage squeeze on female and the limitation of unmarried birth. Generally speaking, the ratio of male and female in an overall community is trend to be equal, and the polyandry policies will inevitably results female overabundance who chooses unmarried birth from the consideration of sexual needs and pension livelihood. From the aspect of religious tradition, firstly, due to Tibetan universally belief in Tibetan Buddhism and more male being monks, which both reduced the social labor productivity, and decreased the reproduction of population, second, a few marriageable males caused the sex ratio imbalanced and remains more marriageable females, resulting unmarried birth phenomenon. From the perspective of outside marriage, although the intermarriage ratio is gradually rising with the development of transportation, ethic fusion, etc. the development of intermarriage is still in a low level which couldn't fundamentally solving the female overabundance problem thus couldn't avoid unmarried birth problem.

Unmarried birth is the inefficient choice of Tibetan women when facing survival pressures. Human activity is restricted by nature all the time. To a large extent, the history of human society development is also a history of living together with nature. Any kind of ancient ethic culture is linked with certain natural environment and nurture and forming in it. When facing the harsh climate conditions and bleak natural environment, the amount of labor become an important guarantee for survival in the premise of outside world can't provide an effective system to supply, especially for Tibetan single females who is facing marriage squeezing and the demand of labor is more urgent, thus unmarried birth became an effective path and means to increase the labor forces. This is a survival strategy to face the harsh realities to some extent. However, the process of a new born turned into labor needs to experience the pure consumer stage, which is serious lagged behind timely. A single female needs a long-time cost to support a new-born growing to a self-support labor, and in the condition without an effective guarantee, they need to pay more. Therefore, the function 
of the idea that single female try to handling survival stress by unmarried birth cannot be denied completely, but its efficiency is undoubtedly low.

Tibetan traditional cultural of inclusive marriage make space for unmarried births.In "The Scientific Theory of Culture”, British social anthropologist Malinowski points out that, marriage custom is relying on traditional forces and a kind of standard behavior driving community members to obey. In this behavior, the real drive force of human sexuality is the order of custom in their consciousness rather than "natural” physiological impulse. Tibetan women's basic desires of human nature in the non-marital state will be restricted, detained or punished without the acquiescence and tolerance of traditional cultural concepts, and the unmarried birth phenomenon will be hard to occur. On the other hand, the Tibetan society holds a relative tolerant attitude on sex and marriage except taboos, and thus the unmarried birth of female got its soil and space to survival.

The transfer logic of unmarried births and women poverty.So far, there exists different voice on the relationship between unmarried birth and poverty, that is, unmarried birth is the cause of poverty or the consequence of poverty. By the investigation on Tibetan female unmarried birth phenomenon, it can be thought that, the two is reciprocal causation and trends to a vicious cycle, which showed in the non-state interference "poverty-unmarried birth-poverty" infinite loop (fig 1). From the perspective of poverty lead to unmarried birth, in terms of economic status, the unmarried birth Tibetan female mostly born originally in poorer families, while in terms of political status, they generally belongs to descendants of the underclass in pre-modern society, reflecting its class inequality of political and economic.

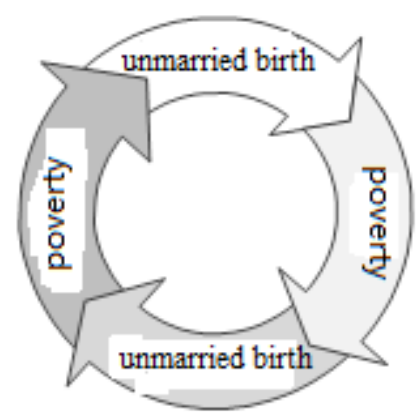

Fig 1 Vicious circle of Tibetan female unmarried birth and poverty

In the relationship of bisexual, female bear more risks and responsibilities and trends to falling in a state of passive and disadvantage. From the perspective of unmarried birth caused poverty, the result of unmarried birth often forming a "mother-child" single parents families. Although both of them can be accepted in Tibetan society without discrimination, their welfare level is often not as good as two-parent families. The female poverty is caused or strengthened by rising unmarried birth children. In the economic analysis of the Tibetan family marriage structure of east Tibetan, Wang Wenchang provided two fresh illustrations of unmarried birth causing female poverty (family poverty).

The poverty caused by unmarried birth is mainly reflected in four aspects. First aspect is economy poverty. Unmarried birth child is mainly raised by their single mother, and in the condition of science and technology haven't being the primary productive force, the manual workers is still the first productivity in Tibet, and the number of workers and the physical strength determines the degree of economic wealth of a family in a large extent. The single-parent family of female unmarried birth in the "mother-child" stage usually presents a state of labor shortage, and couldn't normally act the production and consumption activities as two-parent family is doing, with a fragile ability to prevent risks than other families, thus easily leading the mother and children 
poverty within the family. Next aspect is health poverty. The unmarried birth Tibetan female often work overload in order to survival and rising children, even participate in labor two-three days after delivery (some of them can rest only several hours), which seriously damaged their health, and most of unmarried birth women have poor physical fitness and disease-ridden. Another aspect is development opportunity poverty related with human and social resources upgrade. Tibetan females of single-parent are besieged by poverty due to labor all day and the spare time occupied by heavy low-level activities and narrow living space, which limited their development opportunity of accepting education, enhance human capital and expand networking. The last aspect is emotional poverty. Generally the marriage of unmarried birth mother is difficult, and there appearing some criticism and censure on unmarried birth with the social custom and ethics of the people, causing a great mental stress and personal humiliation, which leading emotional poverty.

\section{Accurate supporting poverty thought and deals with unmarried birth women poverty}

Concepts of accurate supporting poverty.As an important part of the governance system of national poverty reduction, most of the poverty supporting policy is based on the summary, sublimation and promotion of the Guangdong experiences. Its essence is to enable the resources alleviate the poverty of target population and its core content is to achieve "truly supporting poverty, supporting truly poverty”. This idea is the critical inheritance of traditional trickle-down poverty alleviate theory and the broadcast and concrete of the empowering thought of Amartya and other scholars and the concepts of participate anti-poverty and cooperative anti-poverty. And its application is on the basis of identify the female poverty caused by unmarried birth, to institute tailor-precision supporting according to its depth and causes of poverty, enhance their capacity, opportunity and rights of development, and stably out of poverty.

Handling women poverty caused by unmarried births. Despite the legal discussion on unmarried birth, it is a basic fact that the unmarried birth of Tibetan female causing poverty and appearing a vicious circle. No matter it is from the aspect of maintaining social relationship of female healthy and gender equality or the aspect of the practice of accurate supporting poverty and out of poverty, the block of the relations between unmarried birth and female poverty is a project worthy paying attention to and in-depth research. This is not only concerns gender equality and dignity, more related to the forming of a better social prospect values. For this reason, this paper proposed several advices to block the relationships:

First, establish social maintenance policies for unmarried birth children. From the perspective of accurate alleviate poverty, the institute of personalized assistance measures needs based on its causes of poverty and requires of support. We can learn from foreign experiences to institute social upbringing policy for unmarried birth children, which effectively guarantee their survival and development rights and reduce the rising and economic pressure of single mothers at the same time.

Second, fully play the function of traditional customs formed by religious and clan in Tibetan areas. Tibetan Buddhism believers can supply material assistance by donating, strengthen the broadcast of mercy, love and mutual assistance by fully using and mining Buddhism to forming a better social atmosphere. Clan can strengthen its constraint of male's social behavior, identify their responsibilities and obligations on sexuality, marriage and family, and proving production aid for unmarried birth women within clan.

Third, establish an alternative mechanism for children value and enhance female values. Unmarried birth is an excitability choice of Tibetan female when facing marriage squeeze and survival reality, and the reaction mode adapt to the living environment. Construction an economic and social conditions and policy support system which benefit to reduce the occur of unmarried 
birth, establish an alternative mechanism for children value; increase the investment on Tibetan female human capital, and improve their awareness of the negative effects of unmarried birth, enhance their lightweight revenue capacity to solving survival and retirement problem rather than relying on giving birth; increase the broadcast of scientific knowledge of contraception and birth control education and technical support of health care to improve their capacity of self-control reproductive behavior, which could alleviate the possibility of health poverty and self-development ability and opportunity poverty of Tibetan female.

Fourth, strengthen the policy of national integration. Providing efficient institutional arrangements and policy incentives for other ethics and residents' in and out, encouraging exchanges, communication and marriage among different ethics, increase the networking and marriage opportunity of Tibetan female, alleviating the squeeze of less marriageable objects, which is good for reduce unmarried birth and alleviate emotional poverty.

\section{References}

[1] Liu, M. Evaluation and inspiration of European and American women unmarried birth phenomenon. Journal of China Women’s University 2010 (4): 102-108. (In Chinese)

[2] Duncan G J, Hoffman S D. Welfare benefits, economic opportunities, and out-of-wedlock births among black teenage girls. Demography 1990, 27(4): 519-535.

[3] Akerlof G A, Yellen J L, Katz M L. An analysis of Out-of-Wedlock Childbearing in the United States. The Quarterly Journal of Economics, 1996, 111(2): 277-317.

[4] Whitehead M, Burström B, Diderichsen F. Social policies and the pathways to inequalities in health: a comparative analysis of lone mothers in Britain and Sweden. Social Science and Medicine, 2000, 50(2): 255-270.

[5] Zhang, G. Study on polyandry marriage in Tibetan. Journal of Southwest University for Nationalities (Natural Science Edition) 2013 (7): 32-41. (In Chinese)

[6] Xu, D. Traditional Tibetan Reproductive Custom and its Eugenics analysis. Tibetan Studies 2002 (3): 97-103. (In Chinese)

[7] Que, L. Study on the status of women in Amdo Tibetan culture. Minzu University of China, Beijing, 2005:51. (In Chinese)

[8] Zhou, T. Wang. Study on the changes of Tibetan childbearing culture from marriage culture in the process of social transformation-A case study of Qinghai Zhuocang Tibetan. Journal of Lanzhou University (Social Sciences) 2010 38(2): 61-65. (In Chinese)

[9] Lei, G. Study on modern Tibetan marriage and family. Journal of the Central University of Nationalities 2003 30(2): 65-66. (In Chinese)

[10]Wang, W. Economic analysis of eastern Tibet marriage structures of eastern Tibetan families. Tibetan Studies 2000 (2): 56-60. (In Chinese)

[11]Tan, L. Zheng, X. Huang, C. Study of reproductive behavior of Tibetan women in Qinghai remote pastoral areas. Population and Economics 2012 (3): 107-112. (In Chinese)

[12]Feng, M. Marriage and family pattern and women poverty in Tibet. Tianfu New Idea 2000 (S1): 83+89. (In Chinese)

[13]Zha, T. Dynamic analysis of Tibetan marriage squeeze situation. Market and Demographic 
Analysis 2007 13(6): 52-56. (In Chinese)

[14]Chen, C. Study on the marital status of Tibetan women based on the 1990 census data Population Research 1999 23(4): 9-14. (In Chinese)

[15]Ge, L. History and culture of early Tibetan. Beijing, Commercial Press 2006: 16. (In Chinese)

[16]Li, C. Local adaptation: the interpretation dimension of Tibetan special marriage pattern and legal culture. Journal of Tibet University (Social Sciences) 2013 28(4): 135-140. (In Chinese)

[17]Xu, P. Marriage and family in rural Tibet. Sociological Studies 1996 (5): 87-92. (In Chinese)

[18]Basong, Zhuoma. Outline the traditional marriage and childbirth practices and problems of Tibetan women. Ethic-National Studies 1995 (4): 31-35. (In Chinese)

[19]Ge, Z. Xing, C. Accurate supporting poverty: notion, practice and explanation---based on the survey of two Yinchuan villages. Guizhou Social Sciences 2015 (5): 157-163. (In Chinese)

[20]Huang, C. Qin, Z. Discussion on the precise supporting poverty and the construction of national poverty control system. Journal of China Executive Leadership Academy Yan'an 2015 (1): 131-136. (In Chinese)

[21]Nixon L A. The effect of child support enforcement on marital dissolution. Journal of Human Resources, 1997, 32(1): 159-181.

[22]Li, L. Historical evolution and outlook of Tibetan marriage customary law. Guizhou Ethic Studies 2014 35(10): 29-32. (In Chinese) 